\title{
Estimation of Ambient Turbulence Intensity over Complex Terrain
}

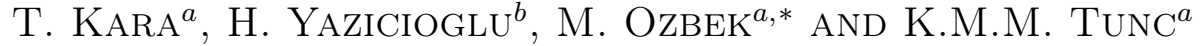 \\ ${ }^{a}$ Istanbul Bilgi University, Faculty of Engineering and Natural Sciences, 34060 Istanbul, Turkey \\ ${ }^{b}$ Technical University of Denmark, Department of Wind Energy, Denmark
}

\begin{abstract}
The aim of the present study is to develop and validate a framework that can handle with ambient flow modeling of operational wind farms over complex terrain. The developed framework estimates the ambient turbulence intensities, which affect the fatigue loads, energy yields and machinery failures, with respect to several incoming flow parameters such as ambient wind speed, altitude and slope angle. Model has been validated with ambient normal turbulence intensities of IEC-61400 standards and reference speed-up models for representative incoming flow conditions, terrain slope angle and altitude based on two wind turbines in the same row that are in the wake of each other for two specific wind direction. The comparison shows fair agreement with the modeled ambient turbulence intensities.
\end{abstract}

DOI: 10.12693/APhysPolA.134.447

PACS/topics: ambient turbulence intensity, complex terrain, slope angle, simple wake models

\section{Introduction}

The complex terrain has considerable influence on the load calculation and power generation in wind power plants. Local wind characteristics are highly dependent on the geometrical shape of the terrain since effective wind direction and turbulence intensities are dominated within. Flow characteristics are in relation with the pressure and temperature levels changing by altitude. Last but not the least, slope angle of terrain changes for various wind directions and it characterizes the flow conditions. Flow separation occurs behind the highest altitude on complex terrain for certain range of slope angle and turbulence intensities that are captured to be much higher comparing with flat terrain conditions. Consequently, rotor is exposed to high wind shear and this influences the accuracy of many linear models. Model accuracy plays a significant role in energy yield and load estimations, since increased turbulence intensity increases the load on the rotor and decreases the power production.

Turbulence intensities are also excited by the interactions among the turbines where flow is disturbed by the rotor. The pressure drop along the stream causes a quick rise on the turbulence intensities behind the rotor. The recovery of the turbulence intensities to ambient levels takes some distance which depends on the rotor diameter and wind speed. Since the disturbed flow cannot recover itself for shorter distances, the flow faces next turbine with higher turbulence intensity than ambient conditions in farm scale. Several engineering models $[1,2]$ are developed for understanding of the major wake characteristics as function of rotor diameter, thrust curve, and ambient turbulence intensity. However, exact ambient turbulence

*corresponding author; e-mail: muammer.ozbek@bilgi.edu.tr intensities can only be measured by the met-mast or the nacelle based sensors. Nacelle based measurements are questionable in lack of met-mast, since the flow is already disturbed by the presence of the rotor. Thus, determination of the actual ambient turbulence intensities for the model inputs is quite important to estimate the actual performance of the wind turbine. Hence, the wind turbulence model is a significant issue on turbine design condition [3]. As mentioned in IEC 61400-1 standard for wind turbine design [4], the ambient turbulence intensities (TI) are represented using suggested parameter values depending on turbine class.

In the present study, a framework is developed and compared in conjunction with simple wake models for estimating the ambient turbulence intensities in wake cases for accurate modeling of operational wind farms over complex terrain. This work is based on the measured 10 min averaged wind speeds on the nacelle and it reveals the interaction between free stream velocities, altitude and slope angle which are key elements of fatigue loads, energy yields, and machinery failures.

\section{Methodology}

Developed framework is based on several classification and filtering operations which are briefly explained in Fig. 1. The framework takes model inputs such as measured atmospheric characteristics. By using simple wake models and wake added turbulence model, the framework is iterated over each wind turbine in wake. Further, the wake superposition of each wake model is found by linear sum formulation. As a result of iteration, reduced free stream wind speed is found and corresponding thrust coefficient (CT) interpolation and iteration over next wind turbine is continued, consequently induced TI is calculated by wake added turbulence model. As a final step, converged ambient TI is saved for the classified ambient conditions. 


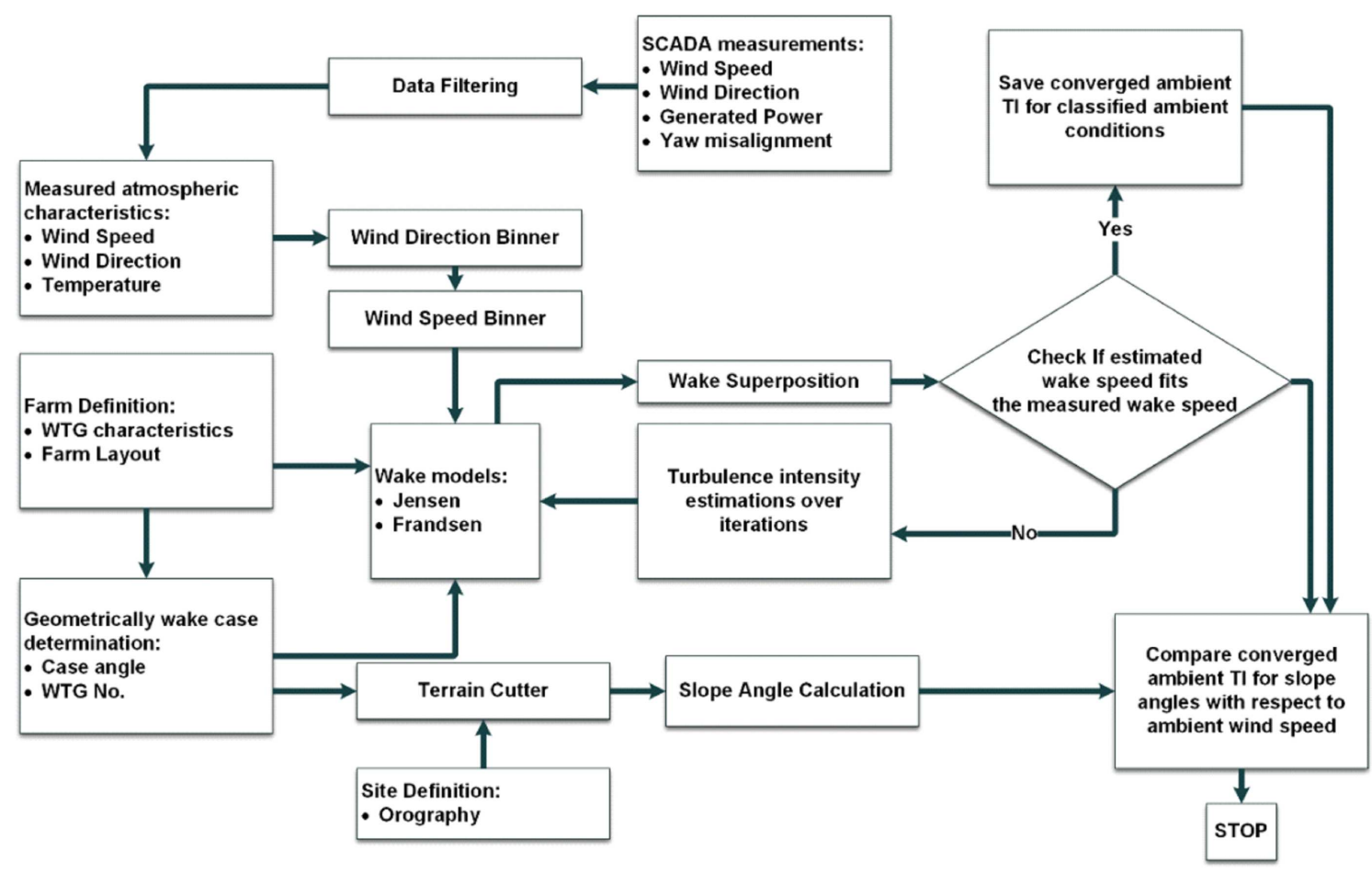

Fig. 1. The framework developed.

The model requires some turbine and site specific inputs such as farm layout, power and thrust curve, horizontal case angle, and wind turbine numbers. This info is also used for sectioning the terrain for specific analyses by calculating terrain slope angles for comparison of converged ambient TI.

Measurements are obtained from SCADA data which are analyzed after two filtering operations. Initially, measurements are binned into certain range of wind direction angle. Latter is binned with respect to averaged wind speed. Termination of the process is done by calibrating ambient turbulence intensities as the model input to meet with measured conditions.

Wake cases are selected due to similar altitude of terrain and slope angle of each terrain from different wind direction of land side and sea side that is flow upstream region. Corresponding parameters to be used in framework is shown in Table I.

Determined wake cases include two turbines in their array at the top of hill ridge (see Fig. 2).

In Fig. 2, the row shows corresponding terrain slope angles for determined turbine arrays. $z$ is the altitude of terrain, $L / D$ is normalized latitude of terrain, $\theta_{s a, \text { Land }}$ is the slope angle of terrain from direction of land side and $\theta_{s a, S e a}$ is the slope angle of terrain from direction of sea side. Lines (red, green and dark blue) represent the each of wake cases.

Two simple wake models are used in this study (e.g. Jensen and Larsen wake model). Jensen wake model is developed by Jensen [2] and further additional developments are done by Katic [5] that are implemented in
TABLE I

Slope angle of terrain from upstream flow/wind direction for each wake case number

\begin{tabular}{c|c|c|c|c}
\hline \hline \multirow{2}{*}{ Analysis parameter } & \multicolumn{2}{|c|}{$\begin{array}{c}\text { Slope angle } \\
\text { of terrain }(\theta)\end{array}$} & \multicolumn{2}{c}{$\begin{array}{c}\text { Wind } \\
\text { direction }[\mathrm{deg}]\end{array}$} \\
\hline Flow upstream & Land & Sea & Land & Sea \\
\hline case 1 & 5.8 & 8.9 & 185 & 5 \\
case 2 & 6.2 & 10.7 & 193 & 13 \\
case 3 & 6.6 & 11.3 & 193 & 13
\end{tabular}

WAsP and WindPRO. Assumption of this wake model is that wake expands linearly with velocity deficit as a function of downstream distance $x$. Horizontal wake deficit at specific downstream section is distributed as top-hat profile based instead of classical single Gaussian shape profile. Model calculates induction factor out of thrust coefficient that is calculated based on incoming velocity to rotor. Wake decay coefficient $k$ has great influence on the wake recovery. Thus, wake deficit is highly dependent on this parameter. Usually it is taken as 0.075 in onshore sites. However, the proposed framework iterates ambient TI and calculates wind speed specific coefficient. Further, it assumes ideal axially symmetric flow and excludes rotation, wake added turbulence and conic shape expansion [6]. Beside the Jensen wake model, recovery of wake is directly manipulated by ambient TI. Pena and Rathmann [7] state that parameter $k$ is a function of height roughness, atmospheric stability, and turbulence separation. Morales [8] formulates wake decay coefficient $k$ as a function of TI. Beside the Jensen wake model, recovery of wake is directly manipulated by ambient TI. 
Larsen wake model was developed and implemented in the WindPRO by Larsen [1]. The model is based on thin shear layer approximation by using Prandtl's turbulent boundary layer equations and the closed form of the Reynolds-averaged Navier-Stokes (RANS). Larsen used the solution of the RANS equations using first and second order approximations and the second order approximation is neglected in last version. Flow is assumed to be incompressible, stationary by neglecting wind shear, thus it is axisymmetric.
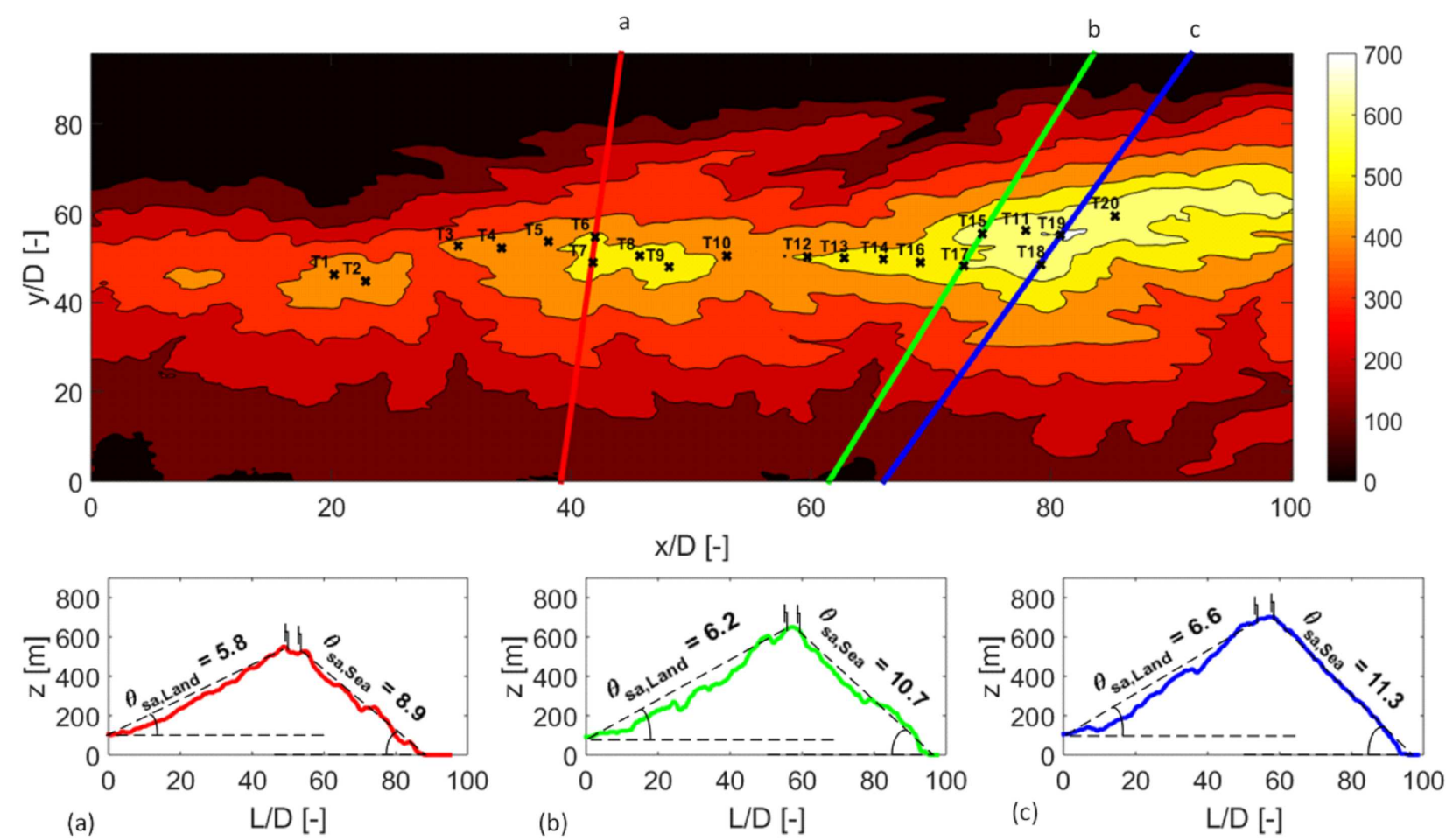

Fig. 2. Contour plot of terrain altitude $y$ normalized with diameter $D$ : (a) cross-section a (red), (b) cross-section b (green), (c) cross-section c (blue).

As the latter step, estimations of each wake models are compared under neutrally stratified flow condition. Multiple wake method is modeled instead of steady state wake growth for developed framework which has been extensively used in the common analytical wind farm models. Dynamic wake meandering impact is neglected since measurements are based on 10 min average periods, though it is not completely realistic to neglect high frequency behaviors of flow. Thus, it is nearly impossible to catch meandering influence of large atmospheric eddies for such frequency range. Many atmospheric characteristics can be easily eliminated by neglecting low frequency part of spectrum.

\section{Results and discussions}

Estimated ambient turbulence intensities are evaluated over complex terrain in conjunction with changing slope angle of terrain. In Fig. 3, $U$ is ambient wind speed and TI is ambient turbulence intensity. First column represents the Jensen wake model (parameterized $k_{w}$ ) according to Pena and Rathmann et al., second column represents the Jensen [2] wake model (parameterized $k_{w}$ ) according to Morales [8], and third column represents the
Larsen wake model [1]. First row, $\theta_{\text {sa,Land }}$ is the slope angle of terrain from direction of land side; second row, $\theta_{s a, \text { Sea }}$, is the slope angle of terrain from direction of sea side.

Consequently, the Jensen wake model is used for two different implementations of wake decay coefficient and all estimations are compared with the Larsen model with respect to incoming velocity. Both three wake models' implementation show fair agreement with modeled ambient turbulence intensities and NTM (see Fig. 3). The highest slope angle of terrain has higher ambient turbulence intensities from direction of land side, although slope angles are fairly similar.

Uncertainty of measurement data can be seen obviously and the fluctuations in first row of Fig. 3 would be caused by flow perturbation, since upstream flow is coming from land terrain. Turbulence intensity is not only affected by heterogeneity of terrain increasingly, but also by the wake of first turbine. In second row of Fig. 3, the trend seems very smooth; there would be no flow perturbation due to homogeneity of terrain, since upstream region is sea. Thus, there can be seen a reverse relation between TI and slope angle of terrain from direction of sea side. 


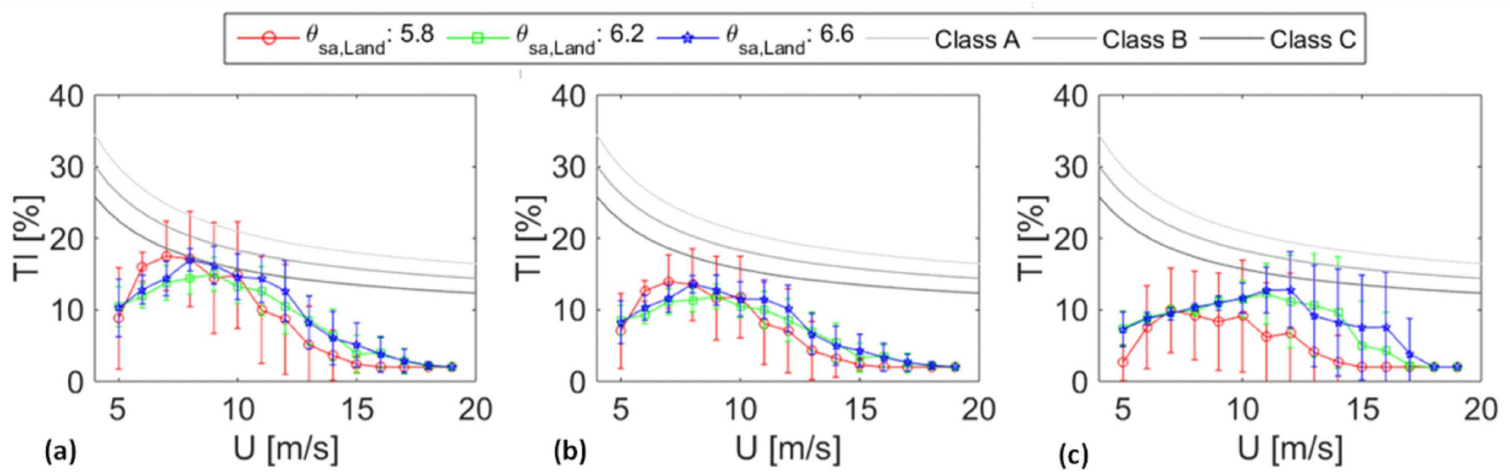

$\multimap \theta_{\text {sa,Sea }}: 8.9 \multimap \theta_{\text {sa,Sea }}: 10.7 \multimap \theta_{\text {sa,Sea }}: 11.3 \multimap$ Class A Class B $\longrightarrow$ Class C
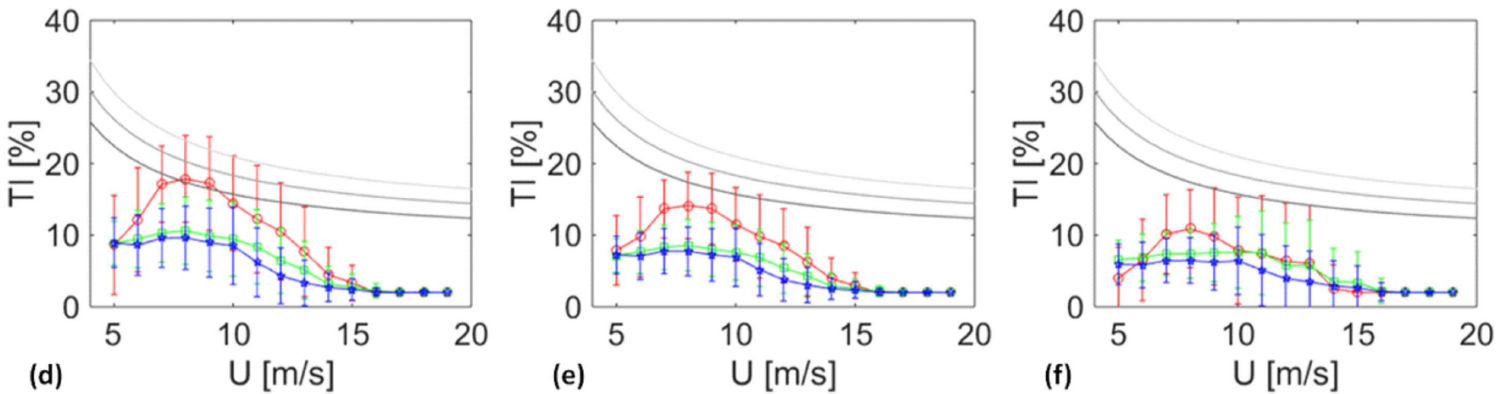

Fig. 3. Comparison of wind speed and ambient turbulence intensity with NTM for each case: (a) wind from land (Pena and Rathmann), (b) wind from land (Morales), (c) wind from land (Larsen), (d) wind from sea (Pena and Rathmann), (e) wind from sea (Morales), (f) wind from sea (Larsen).

Using the formulation of wake decay coefficient in the study of Morales has a better agreement in order to represent ambient turbulence intensity comparing to formulation in study of Pena and Rathmann. Thus, this coefficient is estimated by higher TI values in each case according to other wake models.

\section{Conclusion}

The model in the developed framework has been compared with ambient turbulence intensities of IEC-61400 standards [4] and reference wake models for representative incoming flow conditions, terrain slope angle and altitude based on turbine arrays in the same row that are in the wake of each. Estimations of simple wake models show fair agreement with IEC-61400 standard ambient turbulence intensities.

Consequently, relation between wind speed and turbulence intensity are revealed for complex terrain conditions at different upstream region. Influence of slope angle over wind speed and ambient turbulence intensity relation should be deduced further by much complex techniques and measurements for high resolution frequency analyses.

\section{References}

[1] G.C. Larsen, A Simple Stationary Semi-Analytical Wake Model, Ris $\varnothing-\mathrm{R}-1713(\mathrm{EN})$ Roskilde: Riso National Laboratory, 2009.

[2] N.O. Jensen, A Note on Wind Generator Interaction, Roskilde: Ris $\varnothing-\mathrm{M}, 1983$.

[3] Tzong-Shyng Leu, Jui-Ming Yo, Yi-Ting Tsai, JiuJih Miau, Ta-Chung Wang, Chien-Chou Tseng, Int. J. Mod. Phys. Conf. Series 34, 1460382 (2014).

[4] IEC 61400-1, 3rd ed., International Electrotechnical Commission. International Standard, Wind Turbines - Part 1: Design Requirements, 2005.

[5] I. Katic, J. Højstrup, N.O. Jensen, in: Proc. European Wind Energy Association Conf. and Exhibit., Rome (Italy), 1986, Eds. W. Palz, E. Sesto, Vol. 1, p. 407.

[6] T. Göçmen, P. van der Laan, P.E. Réthoré, A.P. Diaz, G.C. Larsen, S. Ott, Renew. Sustain. Energy Rev. 60, 752 (2016).

[7] A. Peña, O. Rathmann, Wind Energy 17, 1269 (2014).

[8] J.B. Morales, M.Sc. Thesis, Dept. of Earth Sciences, Campus Gotland Uppsala University. 